\title{
Separate common causal explanation and the Bell inequalities
}

\author{
Gábor Hofer-Szabó \\ Department of Logic \\ Eötvös University Budapest \\ Email: gsz@szig.hu
}

\begin{abstract}
In the paper we ask how the following two facts are related: (i) a set of correlations has a local, non-conspiratorial separate common causal explanation; (ii) the set satisfies the Bell inequalities. Our answer will be partial: we show that no set of correlations violating the Clauser-Horne inequalities can be given a local, non-conspiratorial separate common causal model if the model is deterministic.
\end{abstract}

\section{Introduction}

According to the standard interpretation a common causal explanation of a set of EPR correlations consists in providing a so-called common common cause system that is a common screener-off for all correlations of the set such that this common screener-off is local and non-conspiratorial. (For the precise definitions see below.) However, it is well known that the assumption that a set of correlations has a local, non-conspiratorial common common cause system results in various Bell inequalities. Since these Bell inequalities are violated for appropriate measurement settings a common causal explanation of the EPR correlations is excluded - at least according to this interpretation of the common causal explanation.

However, in 1996 Belnap and Szabó came up with a weaker interpretation of the common causal explanation (Belnap, Szabó, 1996). The idea was that a set of correlations may not have a common common cause system but only a set of separate common cause systems explaining the correlations separately. In 2000 Szabó raised the question whether this idea provides a satisfactory common causal explanation for the EPR scenario (Szabó, 2000). To test his idea Szabó took a set of EPR correlations violating the appropriate Bell inequalities and then developed a computer program to generate local, non-conspiratorial separate common cause systems for the given set. The result of the computer simulations was that the chosen set of EPR correlations could be given a local separate common causal explanation, however the common cause systems were conspiratorial in a very tricky way. (See below.) Being unable to remove the unwanted conspiracies Szabó concluded the paper with the conjecture that EPR correlation can not be given a local, non-conspiratorial separate common causal explanation.

Szabó's idea inspired a whole series of papers devoted to the clarification of the possibility of a separate common causal explanation of EPR correlations. In 2005 Grasshoff, Portmann and Wüthrich derived the Wigner-type Bell inequalities from Szabó's premises plus the assumption that the set of correlations consisted of only perfect anticorrelations. (Grasshoff et al, 2005). The assumption of perfect anticorrelations, however, had two unpleasant consequences. First, the fate of the separate common causal explanation of the EPR scenario hinged on a precise experimental test of perfect anticorrelations. Second, the assumption of perfect anticorrelations reduced the separate common 
causal derivation of the Bell inequalities to a standard common common causal derivation. This reduction has been shown by Hofer-Szabó in (Hofer-Szabó, 2008). In the same paper Hofer-Szabó has presented a derivation of Bell inequalities from local, non-conspiratorial separate common causes without assuming perfect anticorrelations. Since a common cause is a special common cause system (a common cause system of size 2) the result was not general enough. In 2007 Portmann and Wüthrich have eliminated the restriction to common causes from the derivation and derived the Clauser-Horne inequality from local, non-conspiratorial separate common cause systems in the context of almost perfect anticorrelations (Portmann and Wüthrich, 2007). Hofer-Szabó generalized this derivation for any $\operatorname{Bell}(\delta)$ inequality that is an inequality differing from some Bell inequality in a term of order of $\delta$ (Hofer-Szabó, 2011). In the light of this derivation a $\delta>0$ threshold could be given for any set of correlations violating the standard Bell inequalities such that if an approriate subset of the original set of correlations differ from perfect anticorrelations less then $\delta$ then the set can not be given a local, non-conspiratorial separate common causal explanation. These results have settled the problem concerning the relation between the separate common causal explanations and the EPR scenario. However, they have not settled the relation between the separate common causal explanations and the Bell inequalities.

On closer examination the strategies used in the papers of the above authors (including the author of the present paper) had a very baffling structure. The reaction of the authors to Szabó's inability to provide a local, non-conspiratorial separate common causal explanation for a set of EPR correlations was the following. The chosen set of correlations cannot have a separate common causal explanation since it violates a Bell inequality which can be derived from the assumption that the given set has a local, non-conspiratorial separate common causal explanation. Of course, the failure of a separate common causal explanation may result from other reasons as well since separate common cause explanations may bring in other constraints between the probability of the correlating events different from the Bell inequalities; still the idea motivating the explanation of this fact was to derive some Bell inequalities from Szabó's premisses. However, it was not that happened. Instead of deriving the appropriate Bell inequality from the assumption that the original set of the correlations chosen by Szabó has a local, non-conspiratorial separate common causal explanation, all the mentioned authors have chosen another set containing only perfect anticorrelations. Then from the assumption that this set of perfect anticorrelations has a local, non-conspiratorial separate common causal explanation they have derived a Bell inequality for the correlations of the original set. So the Bell inequality they reached did not pertain to the original set but to the newly chosen set of perfect anticorrelations.

The effort of all the subsequent papers (Portmann and Wüthrich, 2007), (Hofer-Szabó, 2008) and (Hofer-Szabó, 2011) was to release the strong requirement of perfect anticorrelations in the derivation and to substitute perfect anticorrelations by almost perfect anticorrelations.

Of course, this strategy is impeccable as long as the aim of the proof is to exclude a local, nonconspiratorial separate common causal explanation of the EPR scenario in general. However, it does not explain why Szabó could not provide a local, non-conspiratorial separate common causal explanation for his own set of correlations. Since Szabó's concern was not to give a separate common causal explanation for the perfect anticorrelation set, the violation of Bell inequalities derived from the assumption that the perfect anticorrelation set has a separate common causal explanation did not explain Szabó's failure of providing a separate common causal explanation for his own set. In order to explain this fact one should derive some Bell inequalities from the assumption that Szabó's original set has a local, non-conspiratorial separate common causal explanation.

Here we will provide a partial answer to this problem. We will show that no set of correlations violating the Clauser-Horne inequalities can be given a deterministic, local, non-conspiratorial separate common causal explanation. Since the elimination of the requirement of determinism from the proof is not straightforward, the general question whether correlations violating the Clauser-Horne inequalities can be given a (not necessary deterministic) local, non-conspiratorial separate common 
causal explanation remains open.

In Section 2 we summarize the assumptions of a local, non-conspiratorial common common causal and separate common causal explanation of a set of EPR correlations respectively. In Section 3 we show in sketch the steps how these assumptions result in the Clauser-Horne inequalities if the set for which we are looking for a local, non-conspiratorial separate common causal explanation is a set of perfect or almost perfect anticorrelations. Finally, in Section 4 we drop these extra correlations and derive the Clauser-Horne inequalities from Szabó's original set of correlations for deterministic, local, non-conspiratorial separate common cause systems.

\section{Common causal explanations of EPR correlations}

Consider the Bohm version of the EPR experiment with a pair of spin- $\frac{1}{2}$ particles prepared in the singlet state $\left|\Psi_{s}\right\rangle$. Let $a_{i}$ denote the event that the measurement apparatus is set to measure the spin in direction $\vec{a}_{i}$ in the left wing where $i$ is an element of an index set $I$ of spatial directions; and let $p\left(a_{i}\right)$ stand for the probability of $a_{i}$. Let $b_{j}$ and $p\left(b_{j}\right)$ respectively denote the same for direction $\vec{b}_{j}$ in the right wing where $j$ is again in the index set $I$. (Note that $i=j$ does not mean that $\vec{a}_{i}$ and $\vec{b}_{j}$ are parallel directions.) Furthermore, let $p\left(A_{i}\right)$ stand for the probability that the spin measurement in direction $\vec{a}_{i}$ in the left wing yields the result "up" and let $p\left(B_{j}\right)$ be defined in a similar way in the right wing for direction $\vec{b}_{j}$. According to quantum mechanics the quantum probability of getting "up" in direction $\vec{a}_{i}$ in the left wing; getting "up" in direction $\vec{b}_{j}$ in the right wing; and getting "up" in both directions $\vec{a}_{i}$ and $\vec{b}_{j}$ are given by the following relations

$$
\begin{aligned}
\operatorname{Tr}\left(W_{\left|\Psi_{s}\right\rangle}\left(P_{A_{i}} \otimes I\right)\right) & =\frac{1}{2} \\
\operatorname{Tr}\left(W_{\left|\Psi_{s}\right\rangle}\left(I \otimes P_{B_{j}}\right)\right) & =\frac{1}{2} \\
\operatorname{Tr}\left(W_{\left|\Psi_{s}\right\rangle}\left(P_{A_{i}} \otimes P_{B_{j}}\right)\right) & =\frac{1}{2} \sin ^{2}\left(\frac{\theta_{a_{i} b_{j}}}{2}\right)
\end{aligned}
$$

where $T r$ is the trace function; $W_{\left|\Psi_{s}\right\rangle}$ is the density operator pertaining to the pure state $\left|\Psi_{s}\right\rangle ; P_{A_{i}}$ and $P_{B_{j}}$ denote projections on the eigensubspaces with eigenvalue +1 of the spin operators associated with directions $\vec{a}_{i}$ and $\vec{b}_{j}$ respectively; and $\theta_{a_{i} b_{j}}$ denotes the angle between directions $\vec{a}_{i}$ and $\vec{b}_{j}$.

The standard way to interpret quantum probabilities is to identify them with conditional probabilities as follows:

$$
\begin{aligned}
p\left(A_{i} \mid a_{i} b_{j}\right) & =\operatorname{Tr}\left(W_{\left|\Psi_{s}\right\rangle}\left(P_{A_{i}} \otimes I\right)\right) \\
p\left(B_{j} \mid a_{i} b_{j}\right) & =\operatorname{Tr}\left(W_{\left|\Psi_{s}\right\rangle}\left(I \otimes P_{B_{j}}\right)\right) \\
p\left(A_{i} B_{j} \mid a_{i} b_{j}\right) & =\operatorname{Tr}\left(W_{\left|\Psi_{s}\right\rangle}\left(P_{A_{i}} \otimes P_{B_{j}}\right)\right)
\end{aligned}
$$

where the events $A_{i}, B_{j}, a_{i}$ and $b_{j}(i, j \in I)$ respectively are elements of a classical probability measure space $(X, S, p)$ and the conditional probabilities are defined in the usual way. With this identification quantum mechanics predicts correlation between classical conditional correlations for non-perpendicular directions $\vec{a}_{i}$ and $\vec{b}_{j}$ :

$$
p\left(A_{i} B_{j} \mid a_{i} b_{j}\right) \quad \neq \quad p\left(A_{i} \mid a_{i} b_{j}\right) p\left(B_{j} \mid a_{i} b_{j}\right)
$$

Specially, if the measurement directions $\vec{a}_{i}$ and $\vec{b}_{j}$ are parallel then there is a perfect anticorrelation between the outcomes $A_{i}$ and $B_{i}$ :

$$
p\left(A_{i} B_{j} \mid a_{i} b_{j}\right)=0
$$


A further consequence of (4)-(5) is the so-called surface locality that is for any $i, i^{\prime}, j, j^{\prime} \in I$ the following relations hold

$$
\begin{aligned}
& p\left(A_{i} \mid a_{i} b_{j}\right)=p\left(A_{i} \mid a_{i} b_{j^{\prime}}\right) \\
& p\left(B_{j} \mid a_{i} b_{j}\right)=p\left(B_{j} \mid a_{i^{\prime}} b_{j}\right)
\end{aligned}
$$

Now, let $\left(A_{i}, B_{j}\right)(i, j \in I)$ denote a pair correlating conditionally according to $(7)$ and let $\left\{\left(A_{i}, B_{j}\right)\right\}_{i, j \in I}$ stand for a set of correlating pairs pertaining to the index set $I$. What does a common causal explanation of the set $\left\{\left(A_{i}, B_{j}\right)\right\}_{i, j \in I}$ of correlations consist in? In the following we expose the components of such an explanation.

Let us begin with the definition of the common cause. Let $(X, S, p)$ be a classical probability measure space and let $A$ and $B$ be two (positively) correlating events. Then the common cause of the correlation is the following:

Definition 1. An event $C$ in $S$ is said to be the common cause of the correlation between events $A$ and $B$ only if the events $A, B$ and $C$ satisfy the following relations:

$$
\begin{aligned}
p(A B \mid C) & =p(A \mid C) p(B \mid C) \\
p\left(A B \mid C^{\perp}\right) & =p\left(A \mid C^{\perp}\right) p\left(B \mid C^{\perp}\right) \\
p(A \mid C) & >p\left(A \mid C^{\perp}\right) \\
p(B \mid C) & >p\left(B \mid C^{\perp}\right)
\end{aligned}
$$

where $C^{\perp}$ denotes the orthocomplement of $C$. Equations (11)-(12) are called screening-off properties since conditioning on $C$ and $C^{\perp}$ respectively screens off the correlation between $A$ and $B$. Inequalities (13)-(14) express positive statistical relevance of the cause $C$ on the two effects $A$ and $B$ respectively.

The above definition of the common cause goes back to Reichenbach (Reichenbach, 1956); (although Reichenbach himself did not regard (11)-(14) as a sufficient condition for an event to be a common cause). From the time of Reichenbach's first characterization on the common cause concept has been generalized in two important ways. First, it has been generalized for situations where there are more than one causes present that is for a system of cooperating common causes (Hofer-Szabó, Rédei, 2004, 2006). Second, the inequalities expressing positive statistical relevance have gradually been redarded as being too restrictive and hence have been dropped. As a result the common cause has been characterized simply as a screener-off partition of the algebra defined as follows:

Definition 2. Let again $(X, S, p)$ be a classical probability measure space and let $A$ and $B$ be two correlating events in $S$. Then a partition $\left\{C_{k}\right\}_{k \in K}$ in $S$ is said to be the common cause system of the correlation between events $A$ and $B$ if and only if the following factorization holds for all $k \in K$ :

$$
p\left(A B \mid C_{k}\right)=p\left(A \mid C_{k}\right) p\left(B \mid C_{k}\right)
$$

where $|K|$, the cardinality of $K$ is said to be the size of the common cause system. A common cause system of size 2 is called a common cause.

Definition 2 of the common cause system referred to a single correlation. However, generally we are looking for the causal explanation for a set of correlations. This explanation can be understood in two different ways. Either we provide a separate common cause system for each separate correlation of the given set; or we are looking for a so-called common common cause system that is a partition screening off all correlations of the set. This latter option puts extra requirements on the explanation since it demands that the common cause system pertaining to the different correlations be the same. 
Now, let us apply the concept of common cause systems to EPR correlations. First note that EPR correlations are conditional correlations in the sense of (7) where the conditions represent the choice of the measurement directions. Looking at the spatiotemporal arrengement of the events representing the measurement choices and the measurement outcomes respectively in the opposite wings and the set of events representing the common cause system at the source we can read off the following two spatial separations. The outcome events $A_{i}$ in the left wing are spatially separated from the measurement choice events $b_{j}$ in the right wing; and similarly events $B_{j}$ are spatially separated from events $a_{i}$. The measurement choice events $a_{i}$ and $b_{j}$ are spatially separeted from the events of the common cause system $\left\{C_{k}\right\}$. Turning these two spatiotemporal considerations in statistical relationships we get the so-called locality and no-conspiracy requirements. Applying the above definition of the common cause systems that is the screening-off requirement for conditional probabilities we obtain altogether three demands that a common causal explanation should satisfy. If we demand on the top that the common cause sytem be the same for all correlations of the given set then we arrive at a local, non-conspiratorial common common causal explanation.

Definition 3. Let $\left\{\left(A_{i}, B_{j}\right)\right\}_{i, j \in I}$ be a set of correlating pairs pertaining to the index set $I$ such that $A_{i}, B_{j}, a_{i}$ and $b_{j}$ are elements of a classical probability measure space $(X, S, p)$. Then a local, non-conspiratorial common common causal explanation of the set $\left\{\left(A_{i}, B_{j}\right)\right\}_{i, j \in I}$ consists in providing a partition $\left\{C_{k}\right\}_{k \in K}$ of $S$ such that $\left\{C_{k}\right\}_{k \in K}$ is local, non-conspiratorial and screens off all the correlations of $\left\{\left(A_{i}, B_{j}\right)\right\}_{i, j \in I}$ in the sense that for every $i, i^{\prime}, j, j^{\prime} \in I$ and $k \in K$ the following relations hold:

$$
\begin{aligned}
p\left(A_{i} \mid a_{i} b_{j} C_{k}\right)=p\left(A_{i} \mid a_{i} b_{j^{\prime}} C_{k}\right) & \text { (locality) } \\
p\left(B_{j} \mid a_{i} b_{j} C_{k}\right)=p\left(B_{j} \mid a_{j^{\prime}} b_{j} C_{k}\right) & \text { (locality) } \\
p\left(a_{i} b_{j} C_{k}\right)=p\left(a_{i} b_{j}\right) p\left(C_{k}\right) & \text { (no-conspiracy) } \\
p\left(A_{i} B_{j} \mid a_{i} b_{j} C_{k}\right)=p\left(A_{i} \mid a_{i} b_{j} C_{k}\right) p\left(B_{j} \mid a_{i} b_{j} C_{k}\right) & \text { (screening-off) }
\end{aligned}
$$

On the other hand, if we let the common cause sytem be different for the different correlations of the set then our explanation will be called a local, non-conspiratorial separate common causal explanation.

Definition 4. Let $\left\{\left(A_{i}, B_{j}\right)\right\}_{i, j \in I}$ be a set of correlating pairs pertaining to the index set $I$ such that $A_{i}, B_{j}, a_{i}$ and $b_{j}$ are elements of a classical probability measure space $(X, S, p)$. Then a local, non-conspiratorial separate common causal explanation of the set $\left\{\left(A_{i}, B_{j}\right)\right\}_{i, j \in I}$ consists in finding a separate partition $\left\{C_{k}^{i j}\right\}_{k(i j) \in K(i, j)}$ of $S$ for each correlation in $\left\{\left(A_{i}, B_{j}\right)\right\}_{i, j \in I}$ such that each $\left\{C_{k}^{i j}\right\}_{k(i j) \in K(i, j)}$ is local, non-conspiratorial and screens off the appropriate correlation in $\left\{\left(A_{i}, B_{j}\right)\right\}_{i, j \in I}$ in the sense that for every $i, i^{\prime}, j, j^{\prime} \in I$ and $k(i j) \in K(i, j)$ the following relations hold:

$$
\begin{aligned}
p\left(A_{i} \mid a_{i} b_{j} C_{k}^{i j}\right)=p\left(A_{i} \mid a_{i} b_{j^{\prime}} C_{k}^{i j}\right) & \text { (locality) } \\
p\left(B_{j} \mid a_{i} b_{j} C_{k}^{i j}\right)=p\left(B_{j} \mid a_{j^{\prime}} b_{j} C_{k}^{i j}\right) & \text { (locality) } \\
p\left(a_{i} b_{j} F\right)=p\left(a_{i} b_{j}\right) p(F) & \text { (no-conspiracy) } \\
p\left(A_{i} B_{j} \mid a_{i} b_{j} C_{k}^{i j}\right)=p\left(A_{i} \mid a_{i} b_{j} C_{k}^{i j}\right) p\left(B_{j} \mid a_{i} b_{j} C_{k}^{i j}\right) & \text { (screening-off) }
\end{aligned}
$$

where $F$ in equation (22) is an element of the algebra $S^{\prime} \subset S$ generated by all the elements of every separate common cause system.

To motivate why it is important to demand no-conspiracy (22) in this strong sense namely for any element of the generated algebra and not just for the $C_{k}^{i j}$ elements, recall the tricky conspiracies in Szabó's separate common causal model. As mentioned in the Introduction Szabó was able to 
construct a local separate common causal model for a special set of EPR correlations that was non-conspiratorial in the sense the every $a_{i}$ and $b_{j}$ were independent of every $C_{k}^{i j}$. However, this model was conspiratorial at a deep level - the measurement choices $a_{i}$ and $b_{j}$ correlated with some disjunctions of elements of separate common cause systems such as $C_{k}^{i j} \cup C_{k^{\prime}}^{i^{\prime} j^{\prime}}$. To exclude all these type of conspiracies we demand no-conspiracy in the strong form (22).

Now, we turn to the relation between the local, non-conspiratorial common or separate common causal explanations of the EPR correlations on the one hand and the Bell inequalities on the other.

\section{Bell inequalities}

Now, let us be more specific concerning our set $\left\{\left(A_{i}, B_{j}\right)\right\}_{i, j \in I}$. Let the correlation set consist of four correlating pairs $\left(A_{1}, B_{3}\right),\left(A_{1}, B_{4}\right),\left(A_{2}, B_{3}\right)$ and $\left(A_{2}, B_{4}\right)$. The standard question is usually whether this set can be given a local, non-conspiratorial common common causal explanation in the sense of Definition 3. The answer is well known. $\left\{\left(A_{i}, B_{j}\right)\right\}_{i=1,2 ; j=3,4}$ can be given a local, non-conspiratorial common common causal explanation only if the correlations of the set for any $i, i^{\prime}=1,2 ; j, j^{\prime}=3,4$ and $i \neq i^{\prime}, j \neq j^{\prime}$ satisfy the Clauser-Horne inequalities

$-1 \leqslant p\left(A_{i} B_{j} \mid a_{i} b_{j}\right)+p\left(A_{i} B_{j^{\prime}} \mid a_{i} b_{j^{\prime}}\right)+p\left(A_{i^{\prime}} B_{j} \mid a_{i^{\prime}} b_{j}\right)-p\left(A_{i^{\prime}} B_{j^{\prime}} \mid a_{i^{\prime}} b_{j^{\prime}}\right)-p\left(A_{i} \mid a_{i} b_{j}\right)-p\left(B_{j} \mid a_{i} b_{j}\right) \leqslant 0$

The proof is simple. It is a trivial fact of arithmetic that for any $\alpha, \alpha^{\prime}, \beta, \beta^{\prime} \in[0,1]$ the expression

$$
\alpha \beta+\alpha \beta^{\prime}+\alpha^{\prime} \beta-\alpha^{\prime} \beta^{\prime}-\alpha-\beta
$$

lies in the bound $[-1,0]$. Now let $\alpha, \alpha^{\prime}, \beta, \beta^{\prime}$ be the following conditional probabilities:

$$
\begin{aligned}
\alpha & \equiv p\left(A_{i} \mid a_{i} b_{j} C_{k}\right) \\
\alpha^{\prime} & \equiv p\left(A_{i^{\prime}} \mid a_{i^{\prime}} b_{j^{\prime}} C_{k}\right) \\
\beta & \equiv p\left(B_{j} \mid a_{i} b_{j} C_{k}\right) \\
\beta^{\prime} & \equiv p\left(B_{j^{\prime}} \mid a_{i^{\prime}} b_{j^{\prime}} C_{k}\right)
\end{aligned}
$$

Plugging (26)-(29) into (25) and using locality (16)-(17) one gets that

$$
\begin{array}{r}
-1 \leqslant p\left(A_{i} \mid a_{i} b_{j} C_{k}\right) p\left(B_{j} \mid a_{i} b_{j} C_{k}\right)+p\left(A_{i} \mid a_{i^{\prime}} b_{j} C_{k}\right) p\left(B_{j^{\prime}} \mid a_{i^{\prime}} b_{j} C_{k}\right)+p\left(A_{i^{\prime}} \mid a_{i^{\prime}} b_{j} C_{k}\right) p\left(B_{j} \mid a_{i^{\prime}} b_{j} C_{k}\right) \\
-p\left(A_{i^{\prime}} \mid a_{i^{\prime}} b_{j^{\prime}} C_{k}\right) p\left(B_{j^{\prime}} \mid a_{i^{\prime}} b_{j^{\prime}} C_{k}\right)-p\left(A_{i} \mid a_{i} b_{j} C_{k}\right)-p\left(B_{j} \mid a_{i} b_{j} C_{k}\right) \leqslant 0
\end{array}
$$

Using screening-off (19) one gets that

$$
\begin{array}{r}
-1 \leqslant p\left(A_{i} B_{j} \mid a_{i} b_{j} C_{k}\right)+p\left(A_{i} B_{j^{\prime}} \mid a_{i^{\prime}} b_{j} C_{k}\right)+p\left(A_{i^{\prime}} B_{j} \mid a_{i^{\prime}} b_{j} C_{k}\right) \\
-p\left(A_{i^{\prime}} B_{j^{\prime}} \mid a_{i^{\prime}} b_{j^{\prime}} C_{k}\right)-p\left(A_{i} \mid a_{i} b_{j} C_{k}\right)-p\left(B_{j} \mid a_{i} b_{j} C_{k}\right) \leqslant 0
\end{array}
$$

Finally, multiplying by $p\left(C_{k}\right)$, summing up for the indices $k$ and using no-conspiracy (18) one obtains (24).

An example for a correlation set which violates (24) and hence can not be given a local, nonconspiratorial common common causal explanation is the one Szabó used in his paper (2000). Here the angles $\theta_{a_{i} b_{j}}$ between the directions $\vec{a}_{i}$ and $\vec{b}_{j}$ are set as follows:

$$
\theta_{a_{1} b_{3}}=\theta_{a_{1} b_{4}}=\theta_{a_{2} b_{3}}=\frac{2 \pi}{3} \quad \text { and } \quad \theta_{a_{2} b_{4}}=0
$$


For this choice of the measurement directions there is a conditional correlation for every $\left(A_{i}, B_{j}\right)$ pair $(i=1,2 ; j=3,4)$ :

$$
\begin{array}{ll}
\frac{3}{8}=p\left(A_{1} B_{3} \mid a_{1} b_{3}\right) & \neq p\left(A_{1} \mid a_{1} b_{3}\right) p\left(B_{3} \mid a_{1} b_{3}\right)=\frac{1}{2} \cdot \frac{1}{2} \\
\frac{3}{8}=p\left(A_{1} B_{4} \mid a_{1} b_{4}\right) & \neq p\left(A_{1} \mid a_{1} b_{4}\right) p\left(B_{4} \mid a_{1} b_{4}\right)=\frac{1}{2} \cdot \frac{1}{2} \\
\frac{3}{8}=p\left(A_{2} B_{3} \mid a_{2} b_{3}\right) & \neq p\left(A_{2} \mid a_{2} b_{3}\right) p\left(B_{3} \mid a_{2} b_{3}\right)=\frac{1}{2} \cdot \frac{1}{2} \\
0=p\left(A_{2} B_{4} \mid a_{2} b_{4}\right) & \neq p\left(A_{2} \mid a_{2} b_{4}\right) p\left(B_{4} \mid a_{2} b_{4}\right)=\frac{1}{2} \cdot \frac{1}{2}
\end{array}
$$

Denote this set of correlations by $\left\{\left(A_{i}, B_{j}\right)\right\}_{C H}$. This set violates the Clauser-Horne inequality

$-1 \leqslant p\left(A_{1} B_{3} \mid a_{1} b_{3}\right)+p\left(A_{1} B_{4} \mid a_{1} b_{4}\right)+p\left(A_{2} B_{4} \mid a_{2} b_{4}\right)-p\left(A_{2} B_{4} \mid a_{2} b_{4}\right)-p\left(A_{1} \mid a_{1} b_{3}\right)-p\left(B_{3} \mid a_{1} b_{3}\right) \leqslant 0$

at the upper bound as follows:

$$
\frac{3}{8}+\frac{3}{8}+\frac{3}{8}-0-\frac{1}{2}-\frac{1}{2} \quad \nless \quad 0
$$

Consequently, $\left\{\left(A_{i}, B_{j}\right)\right\}_{C H}$ can not be given a local, non-conspiratorial common common causal explanation.

Now, let us go over to the question whether $\left\{\left(A_{i}, B_{j}\right)\right\}_{C H}$ (or any other correlation set violating the Clauser-Horne inequalities) can have a local, non-conspiratorial separate common causal explanation. As mentioned in the Introduction Szabó was unable to present a local, non-conspiratorial separate common cause model for $\left\{\left(A_{i}, B_{j}\right)\right\}_{C H}$ because of the unwanted conspiracies. The natural intuition towards this fact was that a local, non-conspiratorial separate common causal explanation of the set $\left\{\left(A_{i}, B_{j}\right)\right\}_{C H}$ results in some Bell inequalities-for example in the above Clauser-Horne inequalities - and the violation of these inequalities for the above setting is responsible for the lack of a separate common causal explanation. Thus, the program has been to show up a derivation of some Bell inequalities from the assumption that $\left\{\left(A_{i}, B_{j}\right)\right\}_{C H}$ has four local, non-conspiratorial separate common cause systems satisfying (20)-(23).

Curiously enough, none of the authors has taken this route. Instead of taking the above set and then searching for a derivation of some Bell inequality from the assumption that this set has a local, non-conspiratorial separate common causal explanation they have chosen another set. This set again consisted of the four correlations of $\left\{\left(A_{i}, B_{i}\right)\right\}(i=1,2,3,4)$ for any of which the angle $\theta_{a_{i} b_{i}}$ was set to 0 . In other words, this set was composed of perfect anticorrelations. Denote this set by $\left\{\left(A_{i}, B_{i}\right)\right\}_{P A}$. For the relation between the sets $\left\{\left(A_{i}, B_{j}\right)\right\}_{C H}$ and $\left\{\left(A_{i}, B_{j}\right)\right\}_{P A}$ see Figure 1 where the continuous lines represent the Clauser-Horne correlations and the dotted lines represent the perfect anticorrelations.

Now, the reasoning has run as follows (for the details see (Grasshoff et al. 2005) and (HoferSzabó, 2008)). Suppose that $\left\{\left(A_{i}, B_{i}\right)\right\}_{P A}$ has a local, non-conspiratorial separate common causal explanation that is four local, non-conspiratorial separate common cause systems $\left\{C_{k}^{i i}\right\}_{k \in K(i)}(i=$ $1,2,3,4)$ satisfying $(20)-(23)$. Since $\left\{\left(A_{i}, B_{i}\right)\right\}_{P A}$ consists of only perfect anticorrelations it is easy to show that from assumptions (20)-(23) it follows that for any $i=1,2,3,4$ there exist a vector $\varepsilon^{i i} \in\{0,1\}^{K(i)}$ such that defining $C^{i i}$ and $C^{i i \perp}$ as

$$
C^{i i} \equiv \bigcup_{k \in K(i)} \varepsilon_{k}^{i i} C_{k}^{i i} ; \quad C^{i i \perp} \equiv \bigcup_{k \in K(i)}\left(1-\varepsilon_{k}^{i i}\right) C_{k}^{i i}
$$

the partitions $\left\{C^{i i}, C^{i i \perp}\right\}(i=1,2,3,4)$ will be local, non-conspiratorial separate common causes that is a separate common cause systems of size 2 for the set $\left\{\left(A_{i}, B_{i}\right)\right\}_{P A}$. Moreover, every $\left\{C^{i i}, C^{i i \perp}\right\}$ 


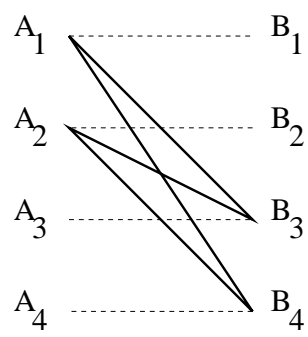

Figure 1: The Clauser-Horne correlation set and the perfect anticorrelation set

will satisfy (20)-(23) deterministically that is each term in (20)-(23) will be either 0 or 1 . Finally, the probability of the separate common causes will equal to the probability of the conditional probabilities $p\left(A_{i} \mid a_{i} b_{i}\right)$ and $p\left(B_{i} \mid a_{i} b_{i}\right)$ :

$$
\begin{aligned}
p\left(C^{i i}\right) & =p\left(A_{i} \mid a_{i} b_{i}\right) \\
p\left(C^{i i \perp}\right) & =p\left(B_{i} \mid a_{i} b_{i}\right)
\end{aligned}
$$

Notice that in this reasoning there has been no mention of the original set $\left\{\left(A_{i}, B_{j}\right)\right\}_{C H}$. How do the correlations of $\left\{\left(A_{i}, B_{j}\right)\right\}_{C H}$ come into the picture?

The joint and marginal conditional probabilities of the Clauser-Horne correlations appear simply using locality (20)-(21) and no-conspiracy (22) for the perfect anticorrelation set. That is for any $i, j=1,2,3,4 ; i \neq j$

$$
\begin{aligned}
p\left(C^{i i}\right) & =p\left(A_{i} \mid a_{i} b_{j}\right) \\
p\left(C^{j j \perp}\right) & =p\left(B_{j} \mid a_{i} b_{j}\right) \\
p\left(C^{i i} C^{j j \perp}\right) & =p\left(A_{i} B_{j} \mid a_{i} b_{j}\right)
\end{aligned}
$$

Now, consider the four events $C^{11}, C^{22}, C^{33 \perp}$ and $C^{44 \perp}$ in $S$. For these events the following simple probabilistic constraint applies:

$$
-1 \leqslant p\left(C^{11} C^{33 \perp}\right)+p\left(C^{11} C^{44 \perp}\right)+p\left(C^{22} C^{33 \perp}\right)-p\left(C^{22} C^{44 \perp}\right)-p\left(C^{11}\right)-p\left(C^{33 \perp}\right) \leqslant 0
$$

Subtituting the probabilities of (43) by the conditional probabilities of (40)-(42) we get the ClauserHorne inequality (35) for the correlation set $\left\{\left(A_{i}, B_{j}\right)\right\}_{C H}$. Since for the measuring setup (30) this inequality is violated there can be given no local, non-conspiratorial separate common causal explanation of the perfect anticorrelation set $\left\{\left(A_{i}, B_{j}\right)\right\}_{P A}$ !

To put is briefly, the necessary condition for $\left\{\left(A_{i}, B_{j}\right)\right\}_{P A}$ to have a local, non-conspiratorial separate common causal explanation is that $\left\{\left(A_{i}, B_{j}\right)\right\}_{C H}$ satisfies the Clauser-Horne inequality $(35)$.

The papers (Portmann and Wüthrich, 2007) and (Hofer-Szabó, 2008, 2011) have repeated the same argumentation for almost perfect anticorrelations. Here we sketch the argument of (Hofer-Szabó, 2011). Consider again a set consisting of four correlating pairs $\left\{\left(A_{i}, B_{i}\right)\right\}_{i=1,2,3,4}$ and suppose that for any $i=1,2,3,4$ the angle $\theta_{a_{i} b_{i}}$ between the measurement choices is such that

$$
\left|\pi-\theta_{a_{i} b_{i}}\right|<2 \arcsin \sqrt{1-2 \delta}
$$

or more simply, let the correlations be such that for any $i=1,2,3,4$

$$
p\left(A_{i} B_{i} \mid a_{i} b_{i}\right) \leqslant \delta
$$


Denote such a set of correlations by $\left\{\left(A_{i}, B_{i}\right)\right\}_{P A(\delta)}$. Again suppose that $\left\{\left(A_{i}, B_{i}\right)\right\}_{P A(\delta)}$ has a local, non-conspiratorial separate common causal explanation. As above, from this assumption it follows that there exist a vector $\varepsilon^{i i} \in\{0,1\}^{K_{i}}$ for any $i=1,2,3,4$ such that defining $C^{i i}$ and $C^{i i \perp}$ as in (37) one get four partitions $\left\{C^{i i}, C^{i i \perp}\right\}_{i=1,2,3,4}$ for which-instead of (38)-(39) - the following inequalities will hold:

$$
\begin{aligned}
\left|p\left(C^{i i}\right)-p\left(A_{i} \mid a_{i} b_{i}\right)\right| & \leqslant 4 \delta \\
\left|p\left(C^{i i \perp}\right)-p\left(B_{i} \mid a_{i} b_{i}\right)\right| & \leqslant 4 \delta
\end{aligned}
$$

Call these partitions quasi common causes since although they are constructed out of the elements of the common cause systems $\left\{C_{k}^{i i}\right\}$ they do not satisfy screening-off (23) (however they satisfy locality (20)-(21) and no-conspiracy (22)).

Now as above, using locality (20)-(21) and no-conspiracy (22) for the set $\left\{\left(A_{i}, B_{i}\right)\right\}_{P A(\delta)}$ we get that for any $i, j=1,2,3,4$

$$
\begin{aligned}
\left|p\left(C^{i i}\right)-p\left(A_{i} \mid a_{i} b_{j}\right)\right| & \leqslant 4 \delta \\
\left|p\left(C^{j j \perp}\right)-p\left(B_{j} \mid a_{i} b_{j}\right)\right| & \leqslant 4 \delta \\
\left|p\left(C^{i i} C^{j j \perp}\right)-p\left(A_{i} B_{j} \mid a_{i} b_{j}\right)\right| & \leqslant 8 \delta
\end{aligned}
$$

Consider again inequality (43) composed of the quasi common causes $C^{11}, C^{22}, C^{33 \perp}$ and $C^{44 \perp}$ and substitute the probabilities of (43) by the conditional probabilities of (48)-(50). Each substitution will cause an error of order of either $4 \delta$ or $8 \delta$. Adding up the errors we obtain the following inequality.

$-1 \leqslant p\left(A_{1} B_{3} \mid a_{1} b_{3}\right)+p\left(A_{1} B_{4} \mid a_{1} b_{4}\right)+p\left(A_{2} B_{3} \mid a_{2} b_{4}\right)-p\left(A_{2} B_{4} \mid a_{2} b_{3}\right)-p\left(A_{1} \mid a_{1} b_{3}\right)-p\left(B_{3} \mid a_{1} b_{3}\right)-40 \delta \leqslant 0(51)$

We refer to this inequlity as a Clauser-Horne $(\delta)$ inequality since (51) differs from the original ClauserHorne inequality (43) in a term of order of $\delta$. Again for the measuring setup (30) the ClauserHorne $(\delta)$ inequality (51) is violated as long as $\delta<\frac{1}{320}$. This excludes a local, non-conspiratorial separate common causal explanation of the almost perfect anticorrelation set $\left\{\left(A_{i}, B_{j}\right)\right\}_{P A(\delta)}$.

This strategy can be generalized for arbitrary Bell $(\delta)$ inequality. In (Hofer-Szabó, 2011) a recipe has been given for deriving any $\operatorname{Bell}(\delta)$ inequality composed of marginal probabilities $p\left(A_{i} \mid a_{i} b_{j}\right)$, $p\left(B_{j} \mid a_{i} b_{j}\right)$ and joint probabilities $p\left(A_{i} B_{j} \mid a_{i} b_{j}\right)$. The recipe is roughly this. Consider a Bell inequality resulting from the local, non-consipratorial common common causal explanation of a set $\left\{\left(A_{i}, B_{j}\right)\right\}$ of correlations. Consider the set $\left\{\left(A_{i}, B_{i}\right)\right\}_{P A(\delta)}$ of almost perfect anticorrelations pertaining to the events $A_{i}$ or $B_{j}$ which appear in either a marginal or a joint probability in the Bell inequality. Suppose that $\left\{\left(A_{i}, B_{j}\right)\right\}_{P A(\delta)}$ has a local, non-conspiratorial separate common causal explanation. This assumption results in a $\operatorname{Bell}(\delta)$ inequality differing from the original Bell inequality in a term of order of $\delta$ where the exact magnitude of this term is the function of the approximation. Choose the setting which violates the Bell inequality maximally. If the $\delta$ term is smaller than the violation of the original Bell inequality than the new $\operatorname{Bell}(\delta)$ inequality will also be violated - excluding a local, nonconspiratorial separate common causal explanation almost perfect anticorrelation set $\left\{\left(A_{i}, B_{j}\right)\right\}_{P A(\delta)}$.

\section{No deterministic, local, non-conspiratorial separate common causal explanation of the Clauser-Horne set}

In the last Section we have posed a question and answered another one. The question was whether the set $\left\{\left(A_{i}, B_{j}\right)\right\}_{C H}$ has a local, non-conspiratorial separate common causal explanation. However, the answer was this. The necessary condition for $\left\{\left(A_{i}, B_{j}\right)\right\}_{P A}$ (or $\left.\left\{\left(A_{i}, B_{j}\right)\right\}_{P A(\delta)}\right)$ to have a local, non-conspiratorial separate common causal explanation is that $\left\{\left(A_{i}, B_{j}\right)\right\}_{C H}$ satisfies the ClauserHorne inequality (24). This answer is perfectly adequate if our intention is to exclude the local, 
non-conspiratorial separate common causal explanation of the EPR scenario as such-as it was the aim of the paper (Grasshoff et al. 2005). But it does not at all explain the fact why Szabó was not able to give a local, non-conspiratorial separate common causal explanation of his original set $\left\{\left(A_{i}, B_{j}\right)\right\}_{C H}$. This latter question can be answered only if we derive some Bell inequalities from the assumption that the original set $\left\{\left(A_{i}, B_{j}\right)\right\}_{C H}$ has a local, non-conspiratorial separate common causal explanation; or we show up other reasons for the failure.

In this Section we give an answer to the original question-a partial answer confined to the deterministic case. The answer is this. $\left\{\left(A_{i}, B_{j}\right)\right\}_{C H}$ can not have a deterministic, local, nonconspiratorial separate common causal explanation since this separate common causal explanation implies the same Clauser-Horne inequalities as the local, non-conspiratorial common common causal explanation.

Proposition 1. Let $\left\{\left(A_{i}, B_{j}\right)\right\}_{i=1,2 ; j=3,4}$ be a set of correlating pairs such that $A_{i}, B_{j}, a_{i}$ and $b_{j}$ are elements of a classical probability measure space $(X, S, p)$. Suppose furthermore that $\left\{\left(A_{i}, B_{j}\right)\right\}_{i=1,2 ; j=3,4}$ has a deterministic, local, non-conspiratorial separate common causal explanation in the sense that there exist a separate partition $\left\{C_{k}^{i j}\right\}_{k(i j) \in K(i, j)}$ of $S$ for each correlation of $\left\{\left(A_{i}, B_{j}\right)\right\}_{i=1,2 ; j=3,4}$ such that $\left\{C_{k}^{i j}\right\}_{k(i j) \in K(i, j)}$ satisties (20)-(23) and $p\left(A_{i} \mid a_{i} b_{j} C_{k}^{i j}\right), p\left(B_{j} \mid a_{i} b_{j} C_{k}^{i j}\right) \in\{0,1\}$ for any $i=$ 1,$2 ; j=3,4$ and $k(i j) \in K(i, j)$. Then for any $i, i^{\prime}=1,2 ; j, j^{\prime}=3,4 ; i \neq i^{\prime}, j \neq j^{\prime}$ the Clauser-Horne inequality (24) follows.

Proof. Consider the separate common cause system $\left\{C_{k}^{i j^{\prime}}\right\}\left(i=1,2 ; j^{\prime}=3,4\right)$ pertaining to the correlation $\left(A_{i}, B_{j^{\prime}}\right)$ and let $K^{\prime}$ denote the set of those indices $k \in K$ for which

$$
p\left(A_{i} B_{j^{\prime}} \mid a_{i} b_{j^{\prime}} C_{k}^{i j^{\prime}}\right)=1
$$

Similarly consider the separate common cause system $\left\{C_{l}^{i^{\prime} j}\right\}\left(i^{\prime}=1,2 ; j=3,4 ; i \neq i^{\prime}, j \neq j^{\prime}\right)$ pertaining to the correlation $\left(A_{i^{\prime}}, B_{j}\right)$ and let $L^{\prime}$ denote the set of those indices $l \in L$ for which

$$
p\left(A_{i^{\prime}} B_{j} \mid a_{i^{\prime}} b_{j} C_{l}^{i^{\prime} j}\right)=1
$$

With the index sets $K^{\prime}$ and $L^{\prime}$ in hand define the following two elements of the algebra generated by the separate common cause systems $\left\{C_{k}^{i j^{\prime}}\right\}$ and $\left\{C_{l}^{i^{\prime} j}\right\}$

$$
\begin{aligned}
& C^{i j^{\prime}} \equiv \bigcup_{k \in K^{\prime}} C_{k}^{i j^{\prime}} \\
& C^{i^{\prime} j} \equiv \bigcup_{l \in L^{\prime}} C_{l}^{i^{\prime} j}
\end{aligned}
$$

Now, since due to locality (20)-(21) for any $k \in K^{\prime}$ and $l \in L^{\prime}$

$$
\begin{aligned}
& p\left(A_{i} \mid a_{i} b_{j} C_{k}^{i j^{\prime}}\right)=1 \\
& p\left(B_{j} \mid a_{i} b_{j} C_{l}^{i^{\prime} j}\right)=1
\end{aligned}
$$

and hence for $C^{i j^{\prime}}$ and $C^{i^{\prime} j}$

$$
\begin{aligned}
& p\left(A_{i} \mid a_{i} b_{j} C^{i j^{\prime}}\right)=1 \\
& p\left(B_{j} \mid a_{i} b_{j} C^{i^{\prime} j}\right)=1
\end{aligned}
$$

it follows that

$$
\begin{aligned}
a_{i} b_{j} C^{i j^{\prime}} & \subseteq A_{i} \\
a_{i} b_{j} C^{i^{\prime} j} & \subseteq B_{j}
\end{aligned}
$$


except for a set of zero measure. From (56)-(57) we obtain that

$$
a_{i} b_{j}\left(C^{i j^{\prime}} \cup C^{i^{\prime} j}\right) \subseteq A_{i} \cup B_{j}
$$

again except for a set of zero measure and hence

$$
p\left(a_{i} b_{j}\left(C^{i j^{\prime}} \cup C^{i^{\prime} j}\right)\right) \leqslant p\left(A_{i} \cup B_{j}\right)
$$

which using no-conspiracy (22) results in

$$
p\left(C^{i j^{\prime}} \cup C^{i^{\prime} j}\right) \leqslant p\left(A_{i} \cup B_{j} \mid a_{i} b_{j}\right)=p\left(A_{i} \mid a_{i} b_{j}\right)+p\left(B_{j} \mid a_{i} b_{j}\right)-p\left(A_{i} B_{j} \mid a_{i} b_{j}\right)
$$

Again, due to locality (20)-(21) from (52)-(53) for any $k \in K^{\prime}$ and $l \in L^{\prime}$ one gets

$$
\begin{aligned}
p\left(B_{j^{\prime}} \mid a_{i^{\prime}} b_{j^{\prime}} C_{k}^{i j^{\prime}}\right) & =1 \\
p\left(A_{i^{\prime}} \mid a_{i^{\prime}} b_{j^{\prime}} C_{l}^{i^{\prime} j}\right) & =1
\end{aligned}
$$

and hence

$$
\begin{aligned}
p\left(B_{j^{\prime}} \mid a_{i^{\prime}} b_{j^{\prime}} C^{i j^{\prime}}\right) & =1 \\
p\left(A_{i^{\prime}} \mid a_{i^{\prime}} b_{j^{\prime}} C^{i^{\prime} j}\right) & =1
\end{aligned}
$$

From (59)-(60) we obtain that

$$
\begin{aligned}
& a_{i^{\prime}} b_{j^{\prime}} C^{i j^{\prime}} \subseteq B_{j^{\prime}} \\
& a_{i^{\prime}} b_{j^{\prime}} C^{i^{\prime} j} \subseteq A_{i^{\prime}}
\end{aligned}
$$

except for a set of zero measure and hence

$$
a_{i^{\prime}} b_{j^{\prime}}\left(C^{i j^{\prime}} C^{i^{\prime} j}\right) \subseteq A_{i^{\prime}} B_{j^{\prime}}
$$

again except for a set of zero measure. From (61) it follows that

$$
p\left(a_{i^{\prime}} b_{j^{\prime}}\left(C^{i j^{\prime}} C^{i^{\prime} j}\right)\right) \leqslant p\left(A_{i^{\prime}} B_{j^{\prime}}\right)
$$

or using no-conspiracy (22)

$$
p\left(C^{i j^{\prime}} C^{i^{\prime} j}\right) \leqslant p\left(A_{i^{\prime}} B_{j^{\prime}} \mid a_{i^{\prime}} b_{j^{\prime}}\right)
$$

Now, from (52)-(53) using the theorem of total probability and no-conspriracy (22) one obtains that

$$
\begin{aligned}
& p\left(C^{i j^{\prime}}\right)=p\left(A_{i} B_{j^{\prime}} \mid a_{i} b_{j^{\prime}}\right) \\
& p\left(C^{i^{\prime} j}\right)=p\left(A_{i^{\prime}} B_{j} \mid a_{i^{\prime}} b_{j}\right)
\end{aligned}
$$

which using the fact that

$$
p\left(C^{i j^{\prime}} \cup C^{i^{\prime} j}\right)=p\left(C^{i j^{\prime}}\right)+p\left(C^{i^{\prime} j}\right)-p\left(C^{i j^{\prime}} C^{i^{\prime} j}\right)
$$

transforms (62) into

$$
p\left(C^{i j^{\prime}} \cup C^{i^{\prime} j}\right) \geqslant p\left(A_{i} B_{j^{\prime}} \mid a_{i} b_{j^{\prime}}\right)+p\left(A_{i^{\prime}} B_{j} \mid a_{i^{\prime}} b_{j}\right)-p\left(A_{i^{\prime}} B_{j^{\prime}} \mid a_{i^{\prime}} b_{j^{\prime}}\right)
$$

Constrasting (58) to (63) we get the Clauser-Horne inequality (24) at the upper bound. To get the inequality at the lower bound just replace $A_{i}$ by $A_{i}^{\perp}$ and follow the steps of the above reasoning. 


\section{Conclusions}

In the paper we addressed the problem as to why Szabó (2000) was unable to yield a local, nonconspiratorial separate common causal model for the EPR scenario. We have shown that the usual answer claiming that the correlation set used by Szabó violates the Clauser-Horne inequalities if we assume that there is a local, non-conspiratorial separate common causal model of another set, is not satisfactory. To explain Szabó's situation one should derive some Bell inequalities from the assumption that there is a local, non-conspiratorial separate common causal model of the original set.

Here we provided a partial answer to this problem. We have shown that no set of correlations violating the Clauser-Horne inequalities can be given a deterministic, local, non-conspiratorial separate common causal explanation. This result was partial since we could not eliminate the requirement of determinism from the proof. So we conclude the paper with the following

Open question: Is it true that no set of correlations violating the Clauser-Horne inequalities can be given a (not necessarily deterministic) local, non-conspiratorial separate common causal explanation? Or in other words, does Proposition 1 hold generally that is without the assumption that $p\left(A_{i} \mid a_{i} b_{j} C_{k}^{i j}\right), p\left(B_{j} \mid a_{i} b_{j} C_{k}^{i j}\right) \in\{0,1\}$ for any $i=1,2 ; j=3,4$ and $k(i j) \in K(i, j)$ ?

Acknowledgements. This work has been supported by János Bolyai Research Scholarship of the Hungarian Academy of Sciences.

\section{References}

Belnap, N., L. E. Szabó (1996). "Branching Space-Time Analysis of the GHZ Theorem," Foundations of Physics, 26, 982-1002.

Grasshoff, G., S. Portmann, A. Wüthrich (2005). "Minimal Assumption Derivation of a Bell-type Inequality," The British Journal for the Philosophy of Science, $\mathbf{5 6}, 663-680$.

Hofer-Szabó, G., M. Rédei (2004). "Reichenbachian Common Cause Systems," International Journal of Theoretical Physics, 34, 1819-1826.

Hofer-Szabó, G., M. Rédei (2006). "Reichenbachian Common Cause Systems of Arbitrary Finite Size Exist," Foundations of Physics Letters, 35, 745-746.

Hofer-Szabó G. (2008). "Separate- versus Common-Common-Cause-Type Derivations of the Bell Inequalities," Synthese, 163/2, 199-215.

Hofer-Szabó G. (2011). "Bell( $\delta$ ) Inequalities Derived from Separate Common Causal Explanation of Almost Perfect EPR Anticorrelations," Foundations of Physics, (forthcoming).

Portmann S., A. Wüthrich (2007). "Minimal Assumption Derivation of a Weak Clauser-Horne Inequality," Studies in History and Philosophy of Modern Physics, 38/4, 844-862.

Reichenbach, H. (1956). The Direction of Time, University of California Press, Berkeley.

Szabó L. E. (2000). "On an Attempt to Resolve the EPR-Bell Paradox via Reichenbachian Concept of Common Cause," International Journal of Theoretical Physics, 39, 911. 